\title{
Detection of Vertical Root Fractures Using Cone-Beam Computed Tomography in the Presence and Absence of Gutta-Percha
}

\author{
Ehsan Hekmatian, ${ }^{1}$ Mitra Karbasi kheir ${ }^{D},^{2}$ \\ Hossein Fathollahzade $\left(D,{ }^{3}\right.$ and Mahnaz Sheikhi ${ }^{4}$ \\ ${ }^{1}$ Dental Implant Research Center, Department of Oral and Maxillofacial Radiology, School of Dentistry, \\ Isfahan University of Medical Sciences, Isfahan, Iran \\ ${ }^{2}$ Department of Oral and Maxillofacial Radiology, School of Dentistry, Islamic Azad University, Isfahan (Khorasgan) Branch, \\ Isfahan, Iran \\ ${ }^{3}$ Department of Oral and Maxillofacial Radiology, School of Dentistry, Isfahan University of Medical Sciences, Isfahan, Iran \\ ${ }^{4}$ Torabinejad Research Center, Department of Oral and Maxillofacial Radiology, School of Dentistry, \\ Isfahan University of Medical Sciences, Isfahan, Iran
}

Correspondence should be addressed to Hossein Fathollahzade; h.f.b1892@gmail.com

Received 3 March 2018; Accepted 12 May 2018; Published 9 July 2018

Academic Editor: Gianluca Plotino

Copyright (C) 2018 Ehsan Hekmatian et al. This is an open access article distributed under the Creative Commons Attribution License, which permits unrestricted use, distribution, and reproduction in any medium, provided the original work is properly cited.

\begin{abstract}
Background. Vertical root fractures (VRFs) can significantly reduce dental prognosis. Cone-beam computed tomography (CBCT) offers better visualization of VRF than conventional radiography. However, gutta-percha creates artifacts in cone-beam CT (CBCT) images and reduces the diagnosis quality. The purpose of this study was to evaluate the accuracy of CBCT in detection of VRF in presence and absence of gutta-percha in canals. Materials and Methods. In this cross-sectional study, 50 extracted mandibular premolars were selected. After preparing the access cavity, canals were instrumented using step-back method, and gutta-percha \#40 was placed afterwards. The fractures were created using electromechanical universal testing machine on 25 teeth. The teeth were randomly placed in dry mandibular dental sockets, and the first CBCTs were taken. Then the gutta-percha was removed, and the second CBCTs were taken. Results. The results of Kappa coefficient between two observers in roots with and without gutta-percha were 0.644 and 0.830 , respectively ( $p$ value $\leq 0.001$ ). The sensitivity and specificity of VRF diagnosis in assessing gutta-percha filled canals were $32 \%$ and $68 \%$ for the first observer, respectively (Kappa $<0.000$, $p$ value $=1.000$ ), and $40 \%$ and $68 \%$ for the second observer, respectively (Kappa $=0.080, p$ value $=0.556)$. The sensitivity and specificity of VRF diagnosis in assessing the empty canals (without gutta-percha) were $72 \%$ and $96 \%$ for the first observer, respectively (Kappa $=0.680, p$ value $\leq 0 / 001$ ), and $72 \%$ and $96 \%$ for the second observer, respectively (Kappa $=0.680, p$ value $\leq 0 / 001)$. Conclusion. The intracanal filling materials such as gutta-percha reduce the diagnostic ability of vertical root fractures. Hence, it is recommended to remove those materials from root canals before imaging to improve the diagnostic potential of CBCT.
\end{abstract}

\section{Introduction}

Vertical root fracture (VRF) occurs along the tooth axis and usually extends from the apex of root to the coronal part and from the internal wall of root canal to the root surface. VRFs may be complete (involving both sides of the root) or incomplete (involving one side of the root). The incidence of VRF in endodontically treated teeth is between 3.7 and
$30.8 \%$. VRF is more prevalent in premolars and mesial root of mandibular molars $[1,2]$. VRF reduces tooth prognosis, can lead to inflammation, followed by bone resorption and granulation tissue formation, and can result in tooth extraction. Identification of VRF is challenging and requires the combination of clinical and radiographic signs and sometimes surgical findings. Overlap of adjacent structures in two-dimensional radiographs (conventional radiography) 
limits the visualization of fracture line. Computed tomography (CT) and cone-beam computed tomography (CBCT), on the other hand, can provide a better visualization of fracture line through multiplanar reconstructed images (axial, coronal, and sagittal planes). However, radiopaque materials like gutta-percha create artifacts in CBCT images due to beam hardening and reduce the diagnostic quality [3-11]. The purpose of this study was to evaluate the accuracy of CBCT in detection of longitudinal root fractures in presence and absence of gutta-percha in canals.

\section{Materials and Methods}

In this cross-sectional study, 50 extracted mandibular premolars were selected and cleaned of calculus, soft tissue, and debris by hand instrumentation. After surface debridement, they were disinfected with $5.25 \%$ sodium hypochlorite $(\mathrm{NaOCl})$. It took two months to collect the extracted teeth. All the teeth were prepared and restored by a single operator to prevent interoperator bias. Standard access cavities were made on them by a cylindrical diamond bur (Tiz Kavan Co., Iran), which was mounted on a high-speed headpiece, using air-water coolant spray (CH-4T5NSK B2/B3, Japan, A1101800). Every four cavities were prepared by a new bur to avoid crack formation. Then, all root canals were instrumented by a circumferential filing technique using hand K-files up to \#40 file (Dentsply Maillefer, Ballaigues, Switzerland) using step-back canal preparation method. Then, a gutta-percha \#40 was placed in each root canal. 25 teeth were included in the control group and 25 teeth were randomly selected for induction of root fracture. The fracture was artificially created using an electromechanical universal testing machine (k-21046, Walter+Bai, Switzerland) with cross-speed of $0.2 \mathrm{~mm} / \mathrm{min}$. The load increased to 500-700 $\mathrm{N}$ until fracture occurred and then the load was immediately stopped as shown by the diagram displayed on the system monitor. All fractured teeth were inspected by a stereomicroscope (Trinocular Zoom Stereo Microscope, SMP 200, HP, USA) at 10× magnification. Stereomicroscope was used as the gold standard to confirm the presence and direction of the fracture line (Figure 1). The other specimens in the control group were also assessed by stereomicroscope to assure that they were fracture-free. The teeth (with and without VRF) were randomly placed in dry mandibular dental sockets. For soft tissue reconstruction, the specimens were placed in a water container, and the first CBCT images were taken. Then the gutta-percha was carefully removed from all root canals to avoid changes in the fracture line/lines, and the second CBCT was taken. CBCT images were taken by Sirona Orthophos, GALILEOS version 1.7, XG 3D (Sirona, Germany). The exposure settings were $85 \mathrm{kVp}, 13 \mathrm{~mA}$, and $5.1 \mathrm{sec}$. The field of view (FOV) of the device was $5 \times 5.5 \mathrm{~cm}$. The images were analyzed by two maxillofacial radiologists on an LG LED computer viewer (E2042C, Korea) using Sidexis IX software. CBCT projections were analyzed in different sectional planes (tangential, cross-sectional, and axial) to detect the presence or absence of VRF, with the possibility to adjust the brightness and contrast and to use the zoom tool (Figures 2 and 3). The observers had been

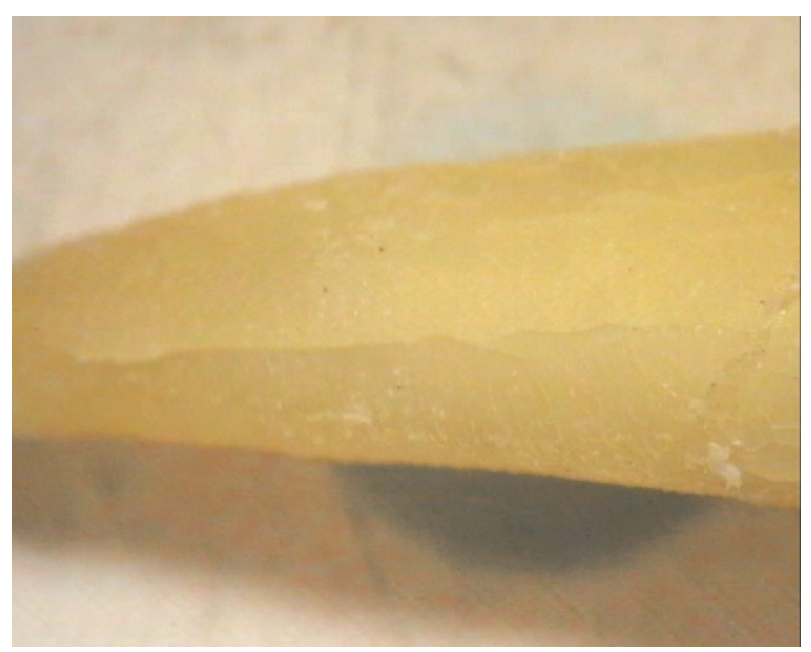

Figure 1: Vertical root fracture under stereomicroscope.

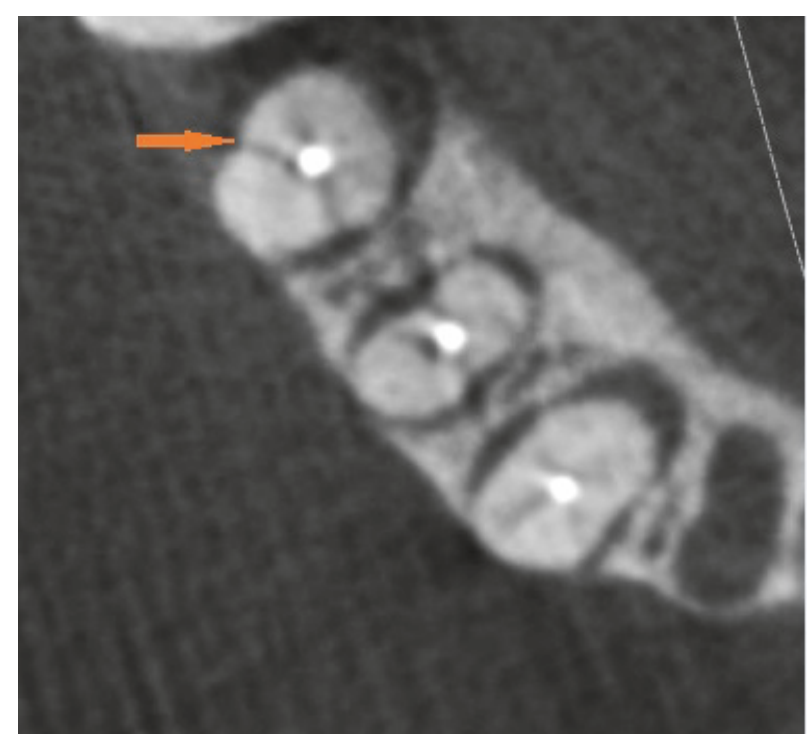

FIGURE 2: CBCT, axial view; red arrow: VRF in gutta-percha filled canals.

calibrated using pilot samples before evaluation of the main samples.

For statistical analysis, the data were analyzed by the Statistical Package for Social Sciences (SPSS) (version 22, SPSS Inc., Chicago, IL). Kappa coefficient was used to analyze the correlation between two observers and to assess the effect of gutta-percha presence on the diagnostic ability of observers.

\section{Results}

The results of Kappa coefficient between two observers in roots with and without gutta-percha were 0.644 and 0.830 , respectively ( $p$ value $\leq 0.001$ ) 
TABLE 1: The frequencies of VRF on CBCT images observed by the first and second observers in control and test groups in gutta-percha filled canals.

\begin{tabular}{|c|c|c|c|c|c|c|c|}
\hline \multicolumn{4}{|c|}{ Observer 1} & \multicolumn{4}{|c|}{ Observer 2} \\
\hline \multirow[t]{2}{*}{ Groups } & \multicolumn{3}{|c|}{ Test } & \multirow[t]{2}{*}{ Groups } & \multicolumn{3}{|c|}{ Test } \\
\hline & Variables & Fracture & No fracture & & Variables & Fracture & No fracture \\
\hline \multirow[t]{2}{*}{ Control } & Fracture & 8 & 17 & Control & fracture & 10 & 15 \\
\hline & No fracture & 8 & 17 & & No fracture & 8 & 17 \\
\hline
\end{tabular}

TABLE 2: The frequencies of VRF on CBCT images observed by the first and second observers in control and test groups in empty canals.

\begin{tabular}{cccccccc}
\hline \multirow{2}{*}{ Groups } & \multicolumn{2}{c}{ Observer 1 } & & \multicolumn{2}{c}{ Observer 2 } \\
\hline \multirow{3}{*}{ Control } & Variables & Fracture & No fracture & & Variables & Fracture & No fracture \\
& Fracture & 18 & 7 & Control & fracture & 18 & 7 \\
& No fracture & 1 & 24 & & No fracture & 1 & 24 \\
\hline
\end{tabular}

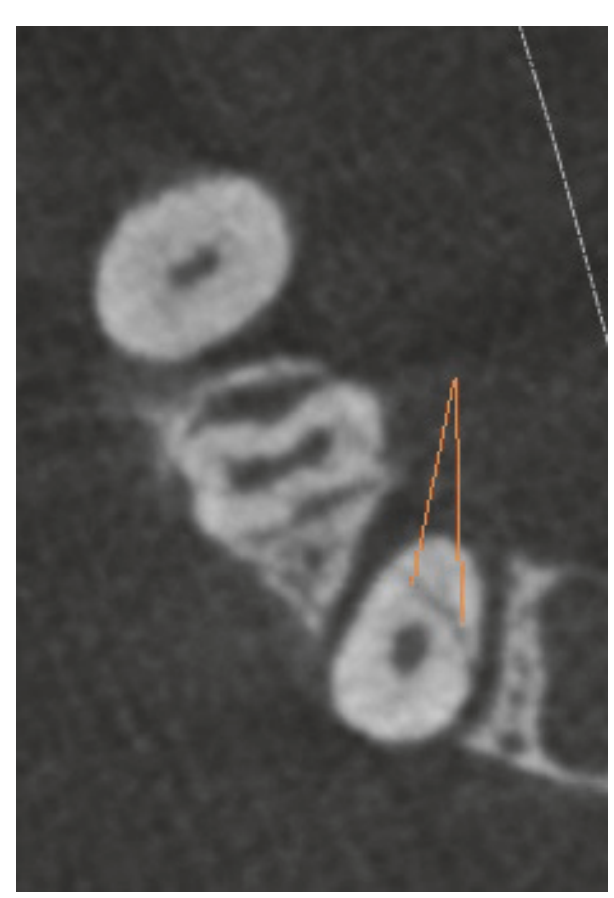

FIgURE 3: CBCT, axial view; red lines: VRF in empty canals (without gutta-percha).

The frequencies of VRF on CBCT images observed by the first and second observers in control and test groups in guttapercha filled canals are shown in Table 1.

The sensitivity and specificity of VRF diagnosis for the first observer in assessing gutta-percha filled canals were $32 \%$ and $68 \%$, respectively (Kappa $<0.000, p$ value $=1.000$ ).

The sensitivity and specificity of VRF diagnosis for the second observer in assessing gutta-percha filled canals were $40 \%$ and $68 \%$, respectively (Kappa $=0 / 080, p$ value $=0 / 556)$.

The frequencies of VRF on CBCT images observed by the first and second observers in control and test groups in empty canals (without gutta-percha) are shown in Table 2.
The sensitivity and specificity of VRF diagnosis for the first observer in assessing empty canals (without guttapercha) were $72 \%$ and $96 \%$, respectively (Kappa $=0 / 680, p$ value $\leq 0 / 001)$.

The sensitivity and specificity of VRF diagnosis for the second observer in assessing empty canals (without guttapercha) were $72 \%$ and $96 \%$, respectively (Kappa $=0 / 680, p$ value $\leq 0 / 001)$.

Assessment of VRF in empty canals (without guttapercha) showed a significant correlation with control samples. However, there was a poor correlation between test and control groups in canals with gutta-percha.

\section{Discussion}

Diagnosis of VRF is important for clinicians because clinicradiographic signs are not completely diagnostic and are similar to other endodontic and periodontal diseases. Failure in VRF diagnosis may lead to surgical procedures or tooth extraction. In this in vitro study, the accuracy of CBCT in detection of VRF in gutta-percha root-filled canals and empty canals (without gutta-percha) was assessed. The presence of radiopaque materials in the root canals, due to beam hardening and creation of radiolucent and radiopaque lines around the radiopaque materials, reduces the diagnostic ability of CBCT [12]. The results of present study showed that the overall sensitivity and specificity of CBCT in detection of VRF in empty canals (without gutta-percha) were significantly higher than those of gutta-percha root-filled canals. In other words, when gutta-percha was placed within root canals, the ability of CBCT in diagnosing VRF was significantly reduced; therefore, gutta-percha can be considered an artifact creating factor (Figure 4).

Our findings were in accordance with those of Neves et al.s study that evaluated the effects of different intracanal materials on the ability of CBCT in VRF detection and found that the presence of gutta-percha had a negative influence on the diagnostic ability of CBCT. In this study, the sensitivity of VRF diagnosis reduced when gutta-percha was 


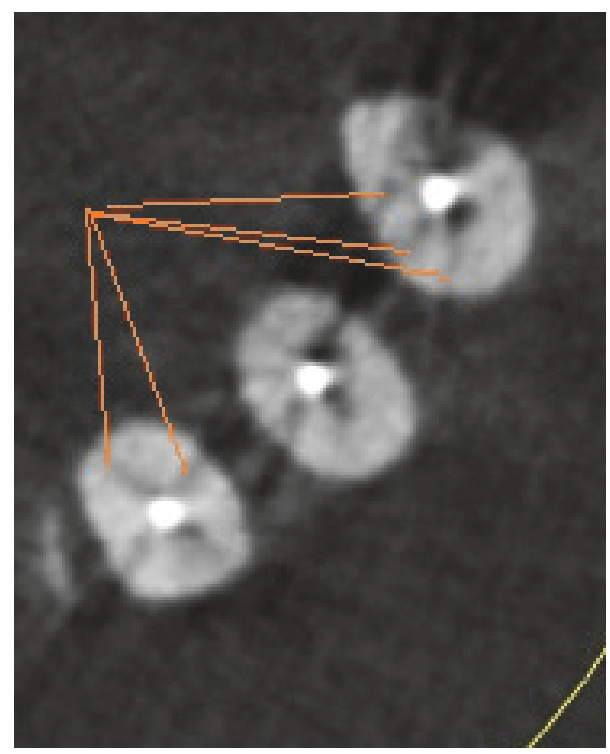

FIGURE 4: Effect of beam hardening on producing artifacts similar to VRF in gutta-percha filled canals.

present in the root canal [4]. Also, the findings confirmed the results of Likubo et al.s study. Their results showed that gutta-percha caused artifacts similar to the fracture lines on CBCT. The difference between our study and that study was consideration of control group, which provided the possibility of measuring the negative effects of guttapercha in the present study [13]. Further, the results of the present research were in agreement with those of Khedmat et al.s study, in which the effect of gutta-percha on diagnosis of vertical root fracture was investigated. In the absence of gutta-percha, CBCT showed a higher diagnostic ability than conventional radiography or even CT scan. However, in the presence of gutta-percha, the diagnostic ability of CBCT was dramatically declined, which is similar to the results of the current study [14].

However, the findings of this study were not in agreement with those of Menezes et al's study that reported no significant differences in VRF detection between absence and presence of gutta-percha in the root canals [15]. The difference between the results of their study with those of the current research can be due to the fact that, in Menezes et al.'s study, the teeth with and without gutta-percha had no similar fractures because of the difference in degree and intensity of fracture. The degree and intensity of vertical root fracture, as confounding factors, can affect the diagnostic ability. In the present study, CBCT was performed on all the fractured and nonfractured teeth filled with gutta-percha. Then, guttapercha was removed from all the root canals, and CBCT was carried out again to eliminate the effect of this confounding factor and ensure that the fracture lines remained constant in the teeth. In other words, in the present study, the degree and intensity of fractures were kept constant in fractured teeth in the presence and absence of gutta-percha. So, this study could be the first attempt to analyze the effect of intracanal filling materials on the diagnostic ability of VRF by eliminating the effect of degree and intensity of fracture as confounding factors.

\section{Conclusion}

The intracanal filling materials such as gutta-percha reduce the diagnostic ability of the vertical root fractures. Hence, it is recommended to remove those materials from root canals before imaging to improve the diagnostic potential of CBCT.

\section{Data Availability}

The data used to support the findings of this study are available from the corresponding author upon request.

\section{Disclosure}

Ehsan Hekmatian and Mitra Karbasi kheir are co-first authors.

\section{Conflicts of Interest}

The authors declare that there are no conflicts of interest regarding the publication of this paper.

\section{Authors' Contributions}

Ehsan Hekmatian and Mitra Karbasi kheir contributed equally to this manuscript.

\section{References}

[1] F. E. Ardakani, S. H. Razavi, and M. Tabrizizadeh, "Diagnostic value of cone-beam computed tomography and periapical radiography in detection of vertical root fracture," Iranian Endodontic Journal, vol. 10, no. 2, pp. 122-126, 2015.

[2] Y. Safi, M. M. Aghdasi, F. Ezoddini-Ardakani, S. Beiraghi, and Z. Vasegh, "Effect of metal artifacts on detection of vertical root fractures using two cone-beam computed tomography systems," Iranian Endodontic Journal, vol. 10, no. 3, pp. 193-198, 2015.

[3] B. Hassan, M. E. Metska, A. R. Ozok, P. van der Stelt, and P. R. Wesselink, "Detection of Vertical Root Fractures in Endodontically Treated Teeth by a Cone Beam Computed Tomography Scan," Journal of Endodontics, vol. 35, no. 5, pp. 719-722, 2009.

[4] F. S. Neves, D. Q. Freitas, P. S. F. Campos, A. Ekestubbe, and S. Lofthag-Hansen, "Evaluation of cone-beam computed tomography in the diagnosis of vertical root fractures: The influence of imaging modes and root canal materials," Journal of Endodontics, vol. 40, no. 10, pp. 1530-1536, 2014.

[5] S. Yiit Özer, "Detection of vertical root fractures by using cone beam computed tomography with variable voxel sizes in an in vitro model," Journal of Endodontics, vol. 37, no. 1, pp. 75-79, 2011.

[6] E. Moudi, S. Haghanifar, Z. Madani, A. Alhavaz, A. Bijani, and M. Bagheri, "Assessment of vertical root fracture using conebeam computed tomography," Imaging Science in Dentistry, vol. 44, no. 1, pp. 37-41, 2014.

[7] L. M. Ferreira, M. A. P. G. Visconti, H. A. Nascimento, R. R. Dallemolle, G. M. Ambrosano, and D. Q. Freitas, "Influence of 
CBCT enhancement filters on diagnosis of vertical root fractures: A simulation study in endodontically treated teeth with and without intracanal posts," Dentomaxillofacial Radiology, vol. 44, no. 5, Article ID 20140352, 2015.

[8] S. A. Khasnis, K. H. Kidiyoor, A. B. Patil, and S. B. Kenganal, "Vertical root fractures and their management," Journal of Conservative Dentistry, vol. 17, no. 2, pp. 103-110, 2014.

[9] S. Kobayashi-Velasco, F. C. S. Salineiro, I. O. Gialain, and M. G. P. Cavalcanti, "Diagnosis of alveolar and root fractures: An in vitro study comparing CBCT imaging with periapical radiographs," Journal of Applied Oral Science, vol. 25, no. 2, pp. 227-233, 2017.

[10] E. Saberi, N. F. Mollashahi, Z. Movasagh, A. A. Moghaddam, and A. Mohammadi, "Value of CBCT in vertical root fracture detection in endodontically-treated teeth," Minerva stomatologica, vol. 66, no. 2, pp. 69-74, 2017.

[11] F. Bragatto, L. Iwaki Filho, A. Kasuya et al., "Accuracy in the diagnosis of vertical root fractures, external root resorptions, and root perforations using cone-beam computed tomography with different voxel sizes of acquisition," Journal of Conservative Dentistry, vol. 19, no. 6, pp. 573-577, 2016.

[12] R. Pauwels, K. Araki, J. H. Siewerdsen, and S. S. Thongvigitmanee, "Technical aspects of dental CBCT: State of the art," Dentomaxillofacial Radiology, vol. 44, no. 1, Article ID 20140224, 2015.

[13] M. Iikubo, T. Osano, T. Sano et al., "Root canal filling materials spread pattern mimicking root fractures in dental CBCT images," Oral Surgery, Oral Medicine, Oral Pathology, Oral Radiology, and Endodontology, vol. 120, no. 4, pp. 521-527, 2015.

[14] S. Khedmat, N. Rouhi, N. Drage, N. Shokouhinejad, and M. H. Nekoofar, "Evaluation of three imaging techniques for the detection of vertical root fractures in the absence and presence of gutta-percha root fillings," International Endodontic Journal, vol. 45, no. 11, pp. 1004-1009, 2012.

[15] R. F. D. Menezes, N. C. D. Araújo, J. M. C. Santa Rosa et al., "Detection of vertical root fractures in endodontically treated teeth in the absence and in the presence of metal post by conebeam computed tomography," BMC Oral Health, vol. 16, no. 1, article no. 48, 2016. 


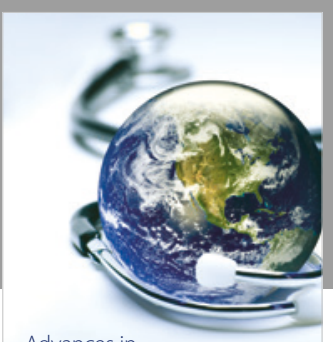

Advances in
Public Health

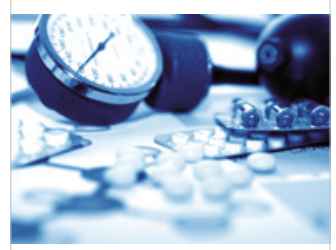

Case Reports in

Medicine

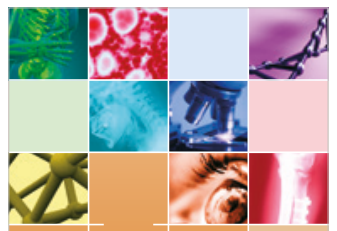

niernational Journal of

Biomaterials
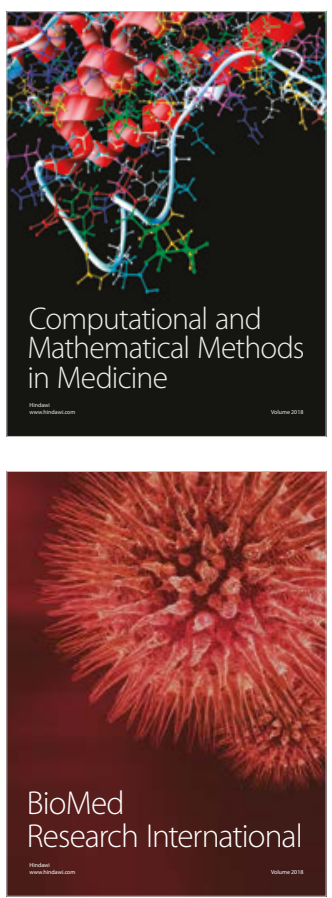

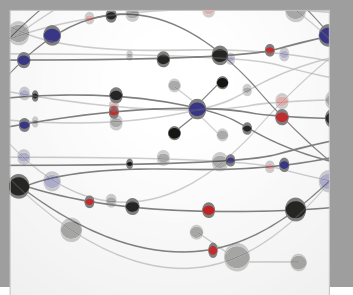

The Scientific World Journal Dentistry

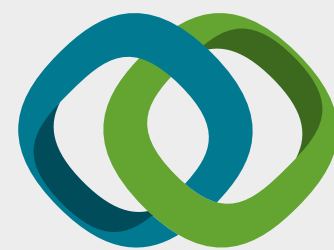

Hindawi

Submit your manuscripts at

www.hindawi.com
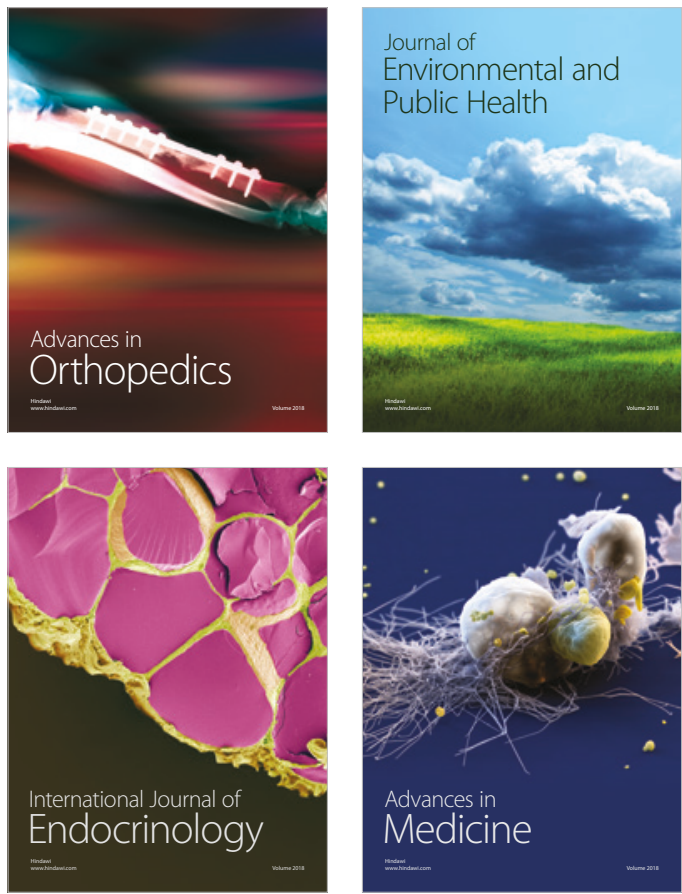
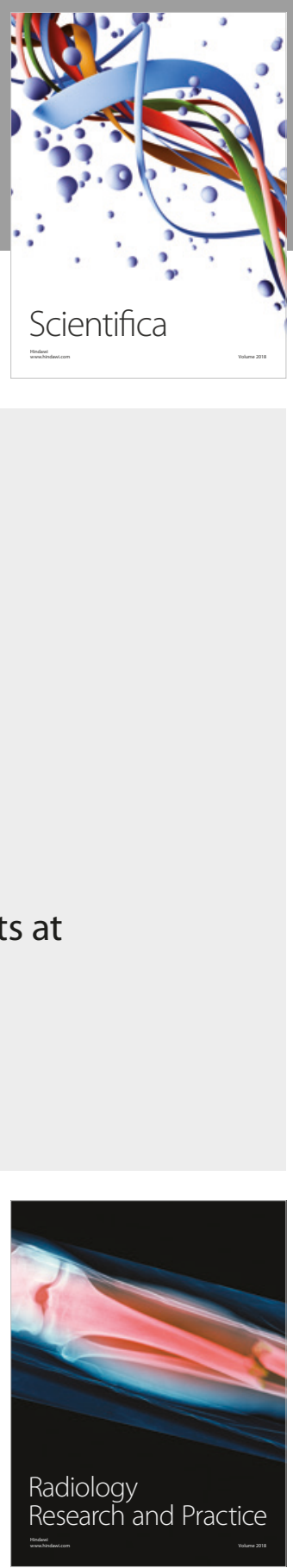

Scientifica

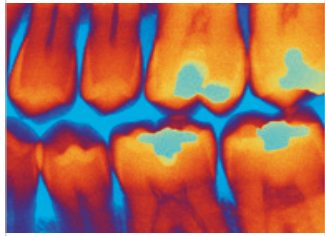

Case Reports in

Dentistry
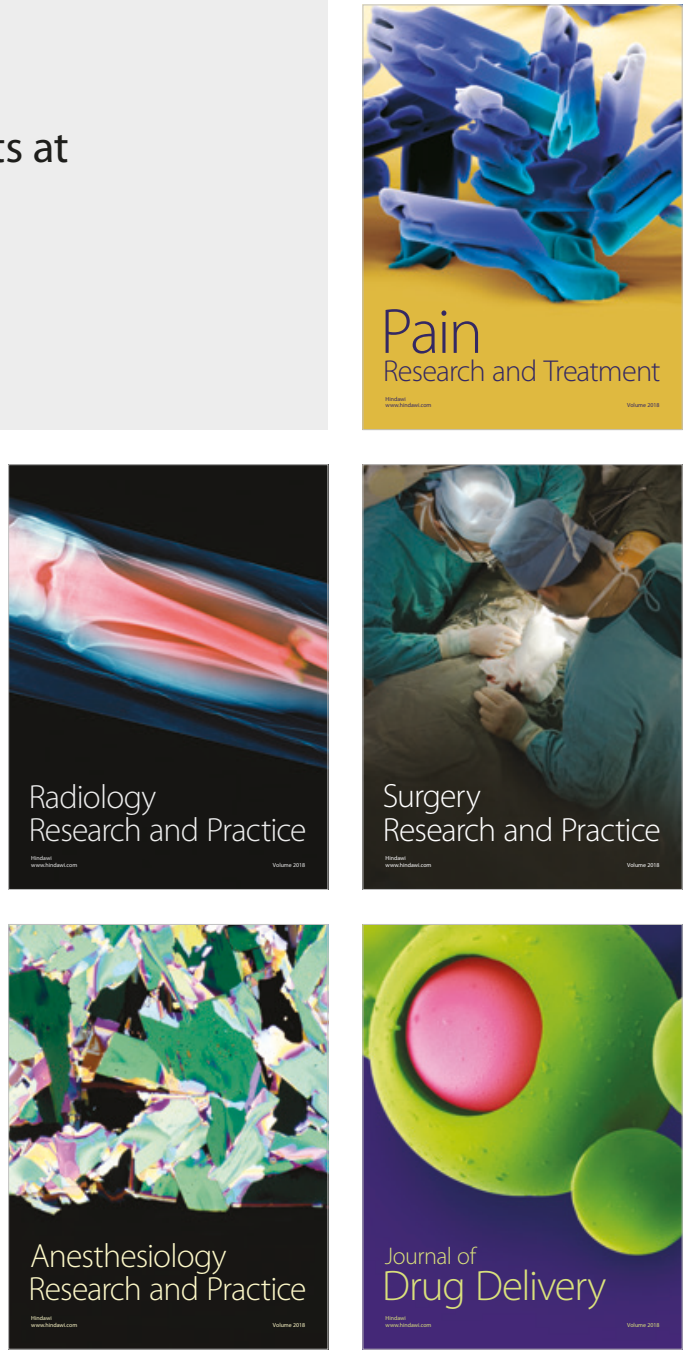